\title{
Potencial de contaminação de solos da região de São José do Rio Preto (SP) pelo herbicida Halossulfurom Metílico.
}

Soil contamination potential of the São José do Rio Preto (SP) region by the herbicide Halossulfuron Methyl.

Potencial de contaminación de suelos de la región de São José do Rio Preto (SP) por el herbicida Halosulfurom Metílico.

Gabriela Byzynski

Dra. Química, UFSCAR, Brasil. Pós doutorando em Química, IQ, UNESP, Brasil. gabi.byzynki@gmail.com

Flávio Fernando Manzini Professor Doutor, UNESP, Brasil. fmanzin@ibilce.unesp.br 


\section{RESUMO}

O halossulfurom metílico é um herbicida comumente utilizado em atividades agrícolas no estado de São Paulo, sendo de vital importância o estudo de sua capacidade de adsorção em solos. O processo de adsorção pode indicar a permanência desse composto no solo ou a contaminação de corpos de água superficiais e subterrâneas. Assim, quatro amostras de solos, de diferentes culturas (horta residencial, milho, cana-de-açúcar, tomate) foram caracterizados em relação aos parâmetros granulométricos e químicos, como $\mathrm{pH}$, teor de matéria orgânica e capacidade de troca de cátions. O comportamento de ionização do herbicida, em diferentes condições de pH, foi determinado, sendo possível determinar o valor do $\mathrm{pK}_{\mathrm{a}}$ do composto. As isotermas do herbicida nos solos apresentam um coeficiente de absorção aparente $\left(K_{d}\right)$ entre 3,66 e 32,86 mL g-1. O herbicida apresenta fraca adsorção na horta e nos solos de cana-de-açúcar e moderada adsorção nos demais solos. A adsorção apresenta correlação negativa com pH e teor de matéria orgânica e correlação positiva com o teor de argila e capacidade de troca de cátions. Assim, é possível que o herbicida seja mais adsorvido nos componentes inorgânicos do solo do que nos orgânicos. Valores de coeficientes de adsorção maiores expressam a permanência no composto no solo por maior tempo. Por outro lado, valores de coeficiente de adsorção menores predizem que o composto pode contaminar águas superficiais e subterrâneas.

PALAVRAS-CHAVE: halossulfurom metílico, isoterma linear, adsorção em solos, análises químicas de solos

\section{ABSTRACT}

Halosulfuron Methyl is an herbicide commonly used in agricultural activities in the São Paulo state, being of vital importance the study of its adsorption capacity in soils. The adsorption process can indicate the permanence of this compound in the soil or the contamination of surface water bodies and groundwater. Thus, four soil samples from different crops (garden, labyrinth, sugar cane, tomato) were characterized in relation to granulometric and chemical parameters, such as $\mathrm{pH}$, organic matter content and cation exchange capacity. The ionization behavior of the herbicide under different $\mathrm{pH}$ conditions was determined and it was possible to define the $p K_{a}$ value of the compound. The herbicide isotherms in soils show an apparent absorption coefficient $\left(K_{d}\right)$ between 3.66 and $32.86 \mathrm{~mL} \mathrm{~g}^{-1}$. The herbicide presents poor adsorption in the garden soil and in the sugarcane soil and moderate adsorption in the other soils. The adsorption presents a negative correlation with $\mathrm{pH}$ and organic matter content and positive correlation with clay content and cation exchange capacity. Thus, it is possible that the herbicide is more adsorbed on inorganic soil components than on organic ones. Higher adsorption coefficients values express the permanence of the compound in the soil for a longer time. On the other hand, lower adsorption coefficient values predict that the compound can contaminate surface and groundwater.

KEY-WORDS: halosulfurom methyl, linear isotherm, soil adsorption, soil chemical analysis

\section{RESUMEN}

El halosulfurom metílico es un herbicida comúnmente utilizado en actividades agrícolas en el estado de São Paulo, siendo de vital importancia el estudio de su capacidad de adsorción en suelos. El proceso de adsorción puede indicar la permanencia de ese compuesto en el suelo o la contaminación de cuerpos de aguas superficiales y subterráneas. Así, cuatro muestras de suelos, de diferentes cultivos (huerta, laberinto, caña de azúcar, tomate) se caracterizaron en relación a los parámetros granulométricos y químicos, como $\mathrm{pH}$, contenido de materia orgánica y capacidad de intercambio de cationes. El comportamiento de ionización del herbicida, en diferentes condiciones de $\mathrm{pH}$, fue determinado, siendo posible determinar el valor del $p K_{a}$ del compuesto. Las isotermas del herbicida en los suelos presentan un coeficiente de absorción aparente $\left(K_{d}\right)$ entre 3,66 y 32,86 mL $\mathrm{g}^{-1}$. El herbicida presenta una débil adsorción en la huerta y en los suelos de caña de azúcar y moderada adsorción en los demás suelos. La adsorción presenta correlación negativa con $\mathrm{pH}$ y contenido de materia orgánica y correlación positiva con el contenido de arcilla y capacidad de intercambio de cationes. Así, es posible que el herbicida sea más adsorbido en los componentes inorgánicos del suelo que en los orgánicos. Los valores de coeficientes de adsorción mayores expresan la permanencia en el compuesto en el suelo por mayor tiempo. Por otro lado, valores de coeficiente de adsorción menores predice que el compuesto puede contaminar aguas superficiales y subterráneas.

PALABRAS-CLAVE: halosulfurom metílico, isoterma lineal, adsorción en suelos, análisis químicos de suelos. 

<smiles>COC(=O)c1c(Cl)nn(C)c1S(=O)(=O)NC(=O)Nc1nc(OC)cc(OC)n1</smiles>

Figura 1: fórmula química estrutural do herbicida halossulfurom metílico

\section{OBJETIVO}

Sabendo que existem poucos estudos sobre a sorção de halossulfurom metílico e a importância deles para a preservação ambiental e a saúde humana, o objetivo deste trabalho é avaliar a sorção do herbicida halossulfurom metílico em quatro diferentes solos brasileiros. A segunda parte deste trabalho é conhecer qual componente do solo é mais importante na adsorção e, para obter esses resultados, a correlação do coeficiente de sorção $\left(\mathrm{K}_{\mathrm{d}}\right)$ com alguns atributos químicos do solo e teor de argila. Os solos utilizados foram os Argissolos da região de São José do Rio Preto, no estado de São Paulo, Brasil, provindos de diferentes culturas horta, milho, canade-açúcar, tomate

\section{METODOLOGIA}

Os reagentes utilizados foram de grau analítico. O halossulfurom metílico foi adquirido de Sigma-Aldrich Riedel-de Häen com uma pureza de $98 \%$ e utilizado sem prévia purificação. Uma solução estoque de $1,90 \times 10^{-3} \mathrm{~mol} \mathrm{~L}^{-1}$ foi preparada em água e usada em todos os experimentos. Amostras com concentrações mais baixas foram obtidas da solução estoque original por diluição.

Quatro amostras de solos de diferentes locais da cidade de São José do Rio Preto, no estado de São Paulo, foram coletadas ( $0-20 \mathrm{~cm}$ de profundidade), classificadas por OLIVEIRA, 1999 como sendo do tipo ultissolos. Estas amostras de solo foram coletadas de diferentes culturas agrícolas: solo 1 (horta residencial); solo 2 (milho); solo 3 (cana de açúcar); solo 4 (tomate) (SILVA, 1999). Estas amostras foram secas ao ar e peneiradas através de uma peneira de $2 \mathrm{~mm}$.

Para o fracionamento granulométrico das amostras de solo foram utilizadas as metodologias de pipetagem e peneiramento sugeridas por SUGUIO, 1973.

A determinação do $\mathrm{pH}$ foi realizada pelo método potenciométrico em solução de cloreto de cálcio (0,01 mol L-1 ) (SILVA, 1999). O teor de matéria orgânica nos solos foi determinado pelo método volumétrico de oxidação, com dicromato de potássio $\left(0,2 \mathrm{~mol} \mathrm{~L}^{-1}\right)$ e sulfato ferroso amoniacal $\left(0,05 \mathrm{~mol} \mathrm{~L}^{-1}\right)$ em meio ácido (CAMARGO et al., 2009). 
A determinação do teor de cálcio e magnésio foi realizada por métodos complexométricos com o uso de EDTA ( $4 \mathrm{~g} \mathrm{~L}^{-1}$ ) e o uso de solução de cloreto de potássio ( $1 \mathrm{~mol} \mathrm{~L}^{-1}$ ) como solução extratora. $O$ teor de alumínio foi determinado pelo método volumétrico por titulação com hidróxido de sódio $\left(0,2 \mathrm{~mol} \mathrm{~L}^{-1}\right)$. $\mathrm{O}$ teor de potássio foi determinado usando solução de extração ácida dupla ( $\mathrm{HCl} \mathrm{0,05} \mathrm{mol} \mathrm{L}^{-1} \mathrm{e} \mathrm{H}_{2} \mathrm{SO}_{4} 0,0125 \mathrm{~mol} \mathrm{~L}^{-1}$ ) e fotometria de chama (SILVA, 1999).

A acidez potencial foi determinada pelo método volumétrico por titulação com acetato de cálcio $\left(0,5 \mathrm{~mol} \mathrm{~L}^{-1}\right)$ como solução extratora e solução de hidróxido de sódio $\left(0,025 \mathrm{~mol} \mathrm{~L}^{-1}\right)$ como titulante, a capacidade de troca catiônica (C.T.C.) foi determinada de acordo à metodologia relatada por (CAMARGO et al., 2009).

O estudo da sorção do halossulfurom metílico nos solos foi realizado, em duplicata, de acordo com o IBAMA, 1990. Soluções de solo foram obtidas pela adição de $10 \mathrm{~mL}$ de soluções de $\mathrm{CaCl}_{2}$ $0,10 \mathrm{~mol} \mathrm{~L}^{-1} \mathrm{em} 1 \mathrm{~g}$ de cada solo. A adição da solução herbicida utilizada variou de 0 a $1,80 \mu \mathrm{mol}$ L-1 (de 0 a $800 \mu \mathrm{g} \mathrm{mL-1)}$. As suspensões foram agitadas a $25^{\circ} \mathrm{C}$ em um agitador horizontal por 24 horas para atingir o equilíbrio. Subsequentemente, as suspensões foram centrifugadas a 9000 rpm por 20 minutos e uma alíquota de $7 \mathrm{~mL}$ de solução sobrenadante foi retirada de cada amostra e filtrada para determinar a concentração de halossulfurom metílico por espectrofotometria UV-visível (Cary 100 Bio-Varian e um cubo de quartzo com maneira de 1 $\mathrm{cm}$ ). A concentração de pesticida adsorvida foi determinada pela diferença da concentração adicionada ao solo e a concentração determinada pelo método analítico através da curva analítica do herbicida na faixa de concentração de 0 a $1,80 \mu \mathrm{mol} \mathrm{L}^{-1}$.

As isotermas de adsorção foram obtidas considerando a sorção como um processo instantâneo, reversível e linear, e o coeficiente de absorção aparente $\left(K_{d}\right)$ foi estimado para cada solo de acordo com:

$$
\mathrm{X} / \mathrm{M}=\mathrm{K}_{\mathrm{d}} \mathrm{C}_{\mathrm{e}}
$$

onde $X / M$ é a quantidade de herbicida adsorvida por grama de solo $\left(\mu \mathrm{g} \mathrm{g}^{-1}\right), \mathrm{C}_{\mathrm{e}}$ é a concentração de equilíbrio do herbicida em solução $\left(\mu \mathrm{g} \mathrm{mL}^{-1}\right)$ e $\mathrm{K}_{\mathrm{d}}$ é o coeficiente de distribuição ou partição do herbicida entre solo e água $\left(\mathrm{mL} \mathrm{g}^{-1}\right)$.

Calculou-se o coeficiente de sorção normalizado pela fração carbono orgânico do solo ( $\left.\mathrm{K}_{\mathrm{oc}}\right)$, através da equação:

$$
\mathrm{K}_{\mathrm{oc}}=\left(\mathrm{K}_{\mathrm{d}} / \mathrm{C}_{\text {org }}\right) \times 100
$$

onde $\mathrm{C}_{\mathrm{org}}$ representa a fração do carbono orgânico do solo.

$O$ teste de Kruskal-Wallis foi utilizado para analisar a variância nos resultados de $K_{d}$. Correlações de Pearson foram também utilizadas entre $K_{o c}, K_{d}$ e alguns parâmetros químicos dos solos amostrados para analisar a correlação entre essas características.

Para os estudos de sorção com variação de $\mathrm{pH}$, utilizou-se ácido sulfúrico (P.A.) para acidificar a solução e as medidas de $\mathrm{pH}$ foram realizadas utilizando o pHmetero ANALION PM608. Todas as soluções foram preparadas com água purificada pelo sistema Mili-Q.

\section{RESULTADOS}


As características químicas e granulométricas dos solos estão apresentadas na Tabela 1 . 0 solo 4 apresentou o menor valor de $\mathrm{pH}(4,66)$ e o solo $1(7,43)$, o maior. Os valores de $\mathrm{pH}$ do solo, entre 5,0 e 6,0, são considerados solos com acidez média como solo $2(5,34)$ e solo $3(5,28)$. Valores superiores a 6,0 são considerados solos com baixa acidez como solo $1(7,43)$ e valores inferiores a 5,0 são considerados solos com alta acidez como solo $4(4,66)$ (TOMÉ, 1997). A presença de íons $\mathrm{Al}^{+3}$ em todas as amostras de solo é confirmada e o baixo valor de $\mathrm{pH}$ pode estar associado à solubilidade dos íons $\mathrm{Al}^{+3}$. À medida que o $\mathrm{pH}$ do solo diminui, o teor de íons $\mathrm{Al}^{+3}$ pode aumentar, causando alguns danos à cultura (TOMÉ, 1997).

Tabela 1. Parâmetros químicos e granulométricos das amostras de solos.

\begin{tabular}{|c|c|c|c|c|}
\hline Atributos & Solo 1 & Solo 2 & Solo 3 & Solo 4 \\
\hline Cultura & Horta residencial & Milho & Cana-de-açúcar & Tomate \\
\hline $\mathrm{pH}$ em $\mathrm{CaCl}_{2}$ & 7,43 & 5,34 & 5,28 & 4,66 \\
\hline \multicolumn{5}{|c|}{$\mathrm{mmol} \cdot \mathrm{dm}^{-3}$} \\
\hline $\mathrm{Ca}^{+2}(\%)$ & 7,60 & 4,00 & 4,90 & 4,20 \\
\hline $\mathrm{Mg}^{+2}(\%)$ & 4,40 & 3,00 & 2,70 & 2,40 \\
\hline $\mathrm{Al}^{+3}(\%)$ & 0,20 & 0,24 & 0,20 & 0,28 \\
\hline $\mathrm{K}^{+}(\%)$ & 0,28 & 0,15 & 0,13 & 0,11 \\
\hline C.T.C. total & 29,61 & 106,98 & 74,56 & 73,54 \\
\hline \multicolumn{5}{|c|}{ g. kg-1 $^{-1}$} \\
\hline M.O. & 34,49 & 16,90 & 14,89 & 14,10 \\
\hline Areia (\%) & 77,90 & 78,70 & 83,60 & 76.40 \\
\hline Silte (\%) & 19,50 & 17,10 & 13,70 & 19,90 \\
\hline Argila (\%) & 2,60 & 4,20 & 2,70 & 3,70 \\
\hline
\end{tabular}

O valor do pH também pode ser correlacionado com o capacidade de troca catiônica total (C.T.C. total), apresentado na Tabela 1, com maior valor para o solo $2(106,98)$, médio para o solo 3 $(74,56)$ e $4(73,54)$, menor valor para o solo $1(29,61)$. A C.T.C. total do solo a $\mathrm{pH} 7,0$ pode ser associado ao número total de cargas negativas que estão disponíveis para o processo de mudança, isto é, lugares ocupados com carga trocável como Ca ${ }^{+2}, \mathrm{Mg}^{+2}, \mathrm{~K}^{+}$e Al ${ }^{+3}$ (KIEHL, 1979). Quanto aos teores de matéria orgânica (M.O.) do solo, o solo 1 apresentou a maior M.O. valor de conteúdo. Além disso, o solo 1 apresenta o valor de $\mathrm{pH}$ superior devido, seguramente, ao mais alto M.O. valor. O solo que exibe M.O. conteúdo maior que $25 \mathrm{~g} \cdot \mathrm{kg}^{-1}$ é considerado com valores elevados de M.O.. O conteúdo dos íons trocáveis também é o mais alto para o solo 1 , porque os solos com M.O.. O conteúdo elevado mostra a capacidade de retenção de importantes cátions e resistência superior na variação de pH. Os demais solos, $1(16,90), 2$ $(14,89), 3(14,10)$, apresentam valores médios de M.O.. 
O teor de argila dos quatro solos é muito baixo e o teor de areia é de alta intensidade. Considerada classificação textural do solo (LEMOS \& SANTOS, 1996), as amostras de solo são classificadas como Areia Franca.

A Figura 2 apresenta o espectro de absorção UV-visível do herbicida em solução com a concentração variando de 0 a $6,34 \times 10^{-5} \mathrm{~mol} \mathrm{~L}^{-1}$. Observa-se que não há variação no comprimento de onda máximo de absorção e a absorção aumenta à medida que a concentração do herbicida aumenta. Este comportamento é um indicativo de que a solução obedece à Lei de Beer-Lambert e é possível determinar a absorção molar $(\varepsilon)$ do herbicida na água. Para esta proposta, foi criado o gráfico da absorbância no comprimento de onda máximo de absorção, $236 \mathrm{~nm}$, em função da concentração de halossulfurom metílico, Figura 2 insert, e é possível confirmar que a solução segue a Lei de Beer-Lambert obtenção de $\varepsilon_{\text {(água) }}=15129 \pm 527 \mathrm{~mol}^{-1} \mathrm{~L}$ $\mathrm{cm}^{-1}$.

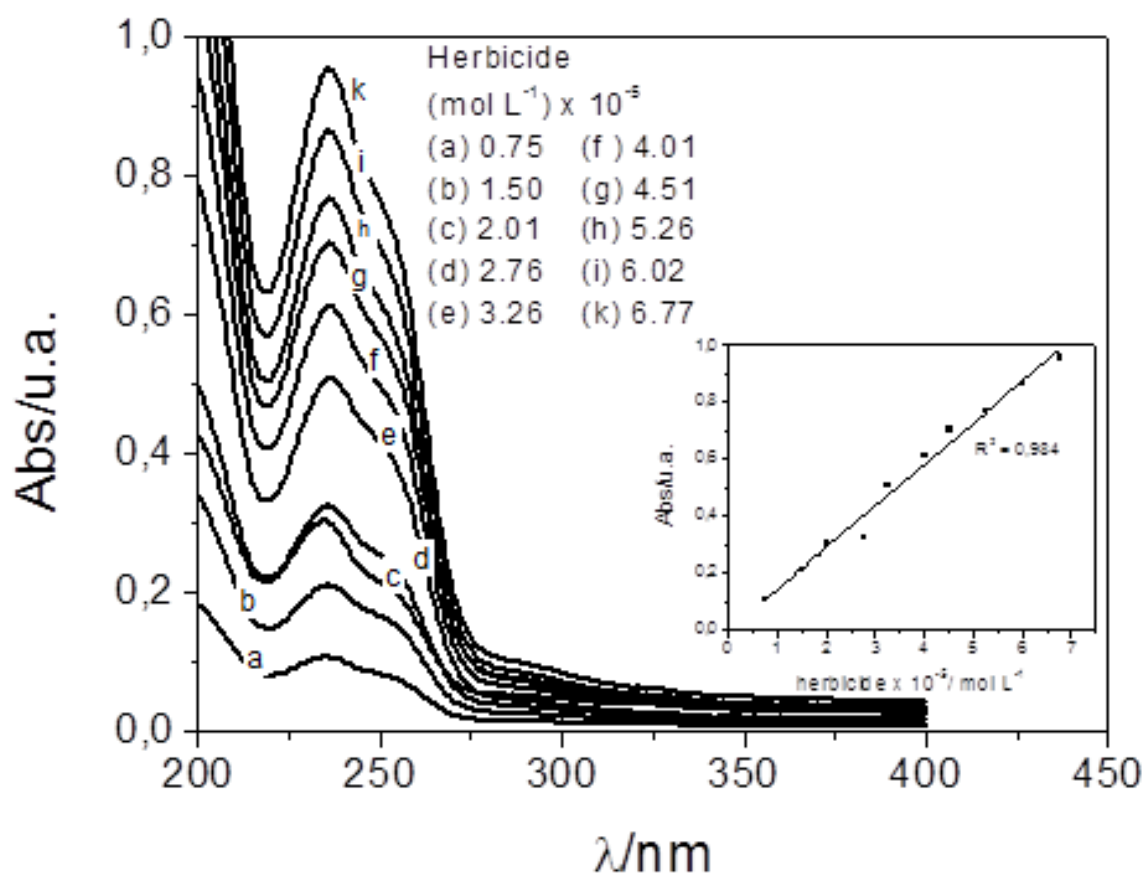

Figura 2: Espectros de absorção UV-vis do herbicida em água com concentrações de 0 a 6,77 x $10^{-5} \mathrm{~mol} \mathrm{~L}^{-1}$, e a relação concentração de Absorbância x Halossulfurom metílico no comprimento máximo de onda de $235 \mathrm{~nm}$.

Para a sorção do herbicida nos solos, a concentração de herbicida utilizada variou de 0 a 1,90 x $10^{-3} \mathrm{~mol} \mathrm{~L}^{-1}$. As suspensões foram agitadas num agitador horizontal durante 24 horas e os sobrenadantes foram analisados. Os espectros de absorção UV-vis dos solos 1, 3 e 4 são mostrados na Figura 3 e para o solo 2 na Figura 4. 
herbicidas. À medida que a concentração do herbicida aumenta, os espectros UV-vis modificam seu comportamento e apresentam maior similaridade com o solo 3, ou seja, é possível notar um "ombro" de absorção em 250 nm à medida que a concentração do herbicida aumenta.
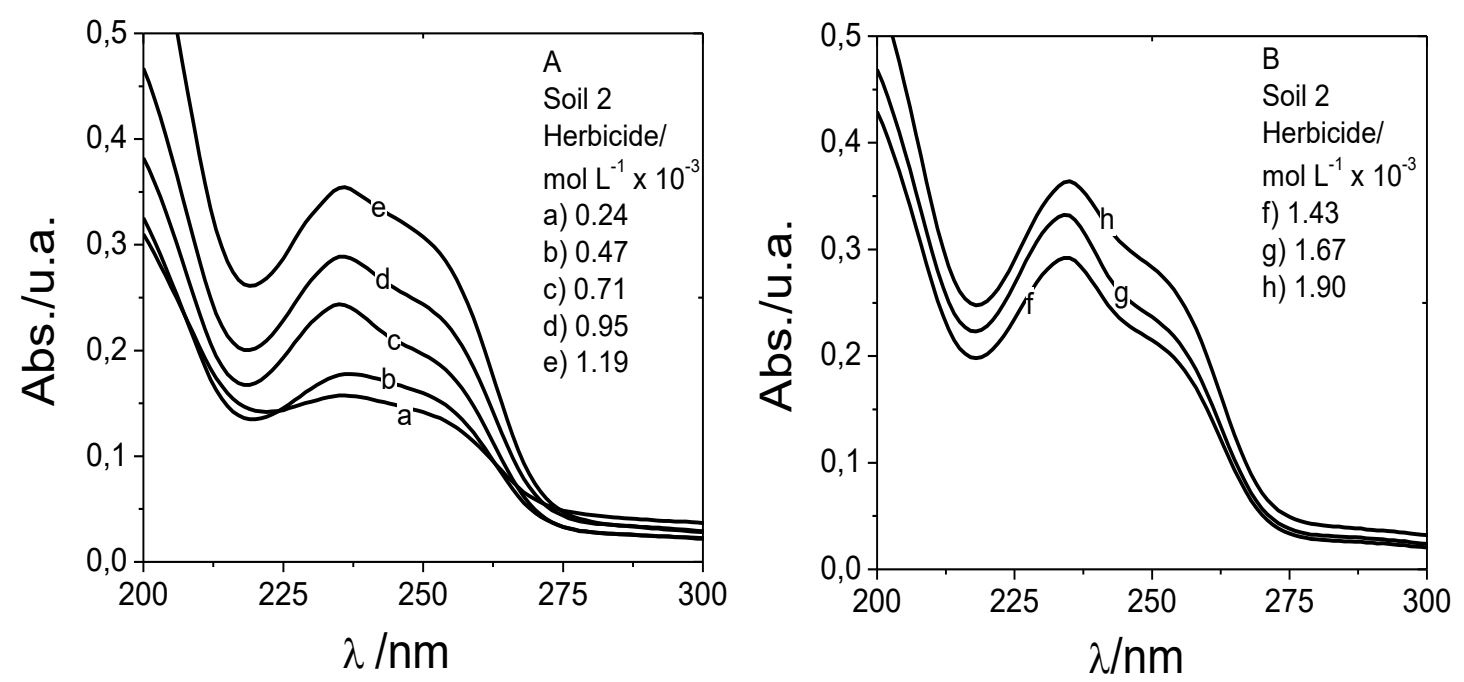

Figura 4: Espectros de UV-vis de soluções herbicidas sobrenadantes no solo 2 (concentração inicial de $A=$ halossulfurom metílico de 0 a $1,18 \times 10^{-3} \mathrm{~mol} \mathrm{~L}^{-1} ; \mathrm{B}=$ concentração inicial de halossulfurom metílico de $1,42 \times 10^{-3}$ $\left.\mathrm{mol} \mathrm{L}^{-1}\right)$.

Para o solo 2, à medida que a concentração da solução aumenta, observa-se a formação da banda de absorção centralizada em 236 nm e um "ombro" de absorção em aproximadamente $250 \mathrm{~nm}$, Figura 4. Os espectros foram divididos em dois gráficos para melhor visualização, devido à concentração a partir de $1,43 \times 10^{-3} \mathrm{~mol} \mathrm{~L}^{-1}$ não apresentar um aumento apreciado na absorção, apenas a melhor definição de banda máxima de absorção em $236 \mathrm{~nm}$.

A variação do comprimento de onda da banda de absorção máxima da solução do solo pode ser correlacionada com o pH em diferentes solos. É possível notar que quando o pH da solução do solo aumenta, o comprimento de onda da banda de absorção máxima muda para valores mais altos. O "ombro" de absorção presente principalmente no solo 2 e 3 também pode estar correlacionado com o pH da solução do solo. A solução do solo 1 tem o pH mais baixo e a presença do "ombro" de absorção não é tão boa observada. Entretanto, quando o pH diminui, como para a solução do solo 4, é possível observar mais claramente o "ombro" de absorção. Esse aparecimento de um "ombro" de absorção pode ser relacionado com a ionização da molécula de halossulfurom metílico em determinado $\mathrm{pH}$. Este comportamento demonstrou que o pH da solução é uma característica importante para a determinação de herbicidas e para melhor entender este comportamento; um estudo com variação de pH na solução herbicida é apresentado. 
indicar a existência de duas espécies no sistema herbicida do solo (possivelmente a forma aniônica e iônica). Este fato pode ser corroborado pelo estudo da variação do pH do herbicida. As isotermas linearizadas do herbicida nos solos são apresentadas na Figura 6. O coeficiente de absorção $\left(K_{d}\right)$ do herbicida no solo, calculado segundo a equação isotérmica, é apresentado na Tabela 2, como também o $K_{o c}$ para cada solo, que é um dos coeficientes que compõem diversos indicadores indicativos de potencial de lixiviação de agrotóxicos. Quanto maior o valor de $\mathrm{K}_{\mathrm{d}}$ apresentado pelo herbicida, maior a tendência de adsorção no solo, consequentemente, menor mobilidade.

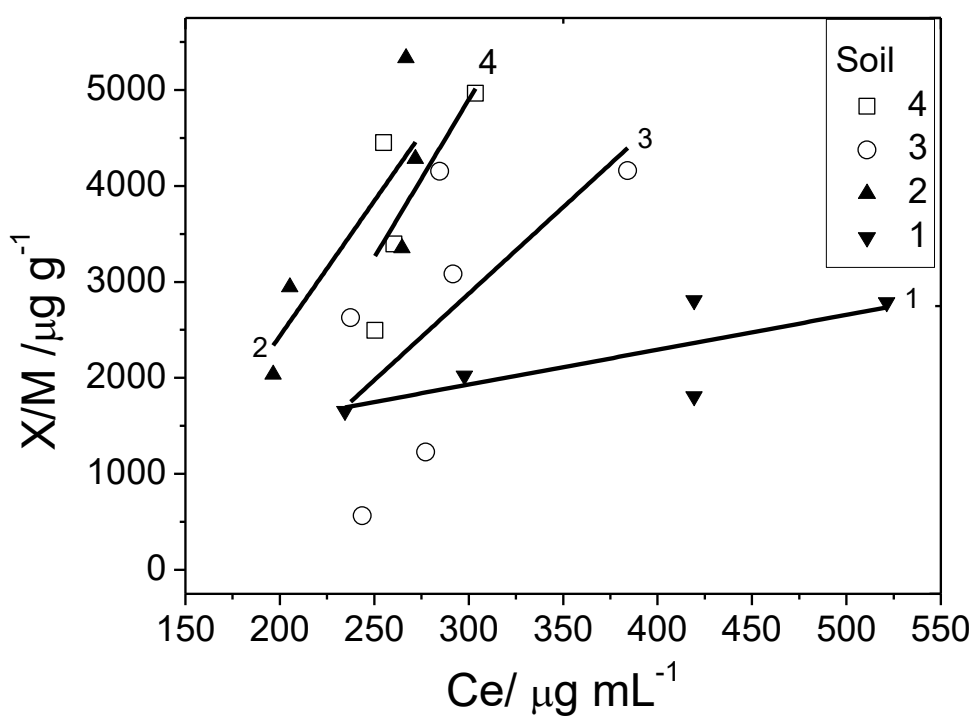

Figura 6. Isotermas de adsorção do halossulfurom metílico nas amostras de solos.

Tabela 2. Valores dos coeficientes $K_{d}$ e $K_{o c}$ do Sistema herbicida-solo.

\begin{tabular}{ccccc}
\hline & Solo 1 & Solo 2 & Solo 3 & Solo 4 \\
\hline$K_{d}\left(\mathrm{~mL} \mathrm{~g}^{-1}\right)$ & 3,63 & 28,15 & 18,03 & 32,86 \\
$\mathrm{~K}_{\mathrm{oc}}\left(\mathrm{mL} \mathrm{g}^{-1}\right)$ & 18,24 & 286,66 & 209,03 & 402,70 \\
\hline
\end{tabular}

Os valores de $K_{d}$ obtidos variam de 3,63 a 32,86 $\mathrm{mL} \mathrm{g}^{-1}$. A partir da análise dos resultados, podese afirmar que o herbicida adsorveu fracamente nos solos $1\left(3,66 \mathrm{~mL} \mathrm{~g}^{-1}\right)$ e $3\left(18,03 \mathrm{~mL} \mathrm{~g}^{-1}\right)$ e apresentou moderada adsorção nos solos $2\left(28,15 \mathrm{~mL} \mathrm{~g}^{-1}\right)$ e $4\left(32,86 \mathrm{~mL} \mathrm{~g}^{-1}\right)$, segundo Oliveira et al [21]. Os valores de $K_{o c}$ apresentaram comportamento semelhante ao da ordem de adsorção, apresentando valores que variam de 18,24 a $402,70 \mathrm{~mL} \mathrm{~g}^{-1}$. O maior valor de $\mathrm{K}_{\mathrm{oc}}$ foi obtido para o solo $4\left(402,70 \mathrm{~mL} \mathrm{~g}^{-1}\right)$ e o menor para o solo $1\left(18,24 \mathrm{~mL} \mathrm{~g}^{-1}\right)$. O coeficiente de distribuição do herbicida $\left(K_{d}\right)$ para cada solo depende das características do solo e a propriedade herbicida. Essa relação pode ser realizada pela correlação de Pearson ( $r$ ), cujos valores 
influência do pH e a M.O. na sorção. O solo 1 apresenta o maior teor de carbono orgânico (19,90 $\mathrm{g} \mathrm{kg}^{-1}$ ) e o maior $\mathrm{pH}(7,43)$, além do menor teor de argila $(2,60 \%)$ e o menor C.T.C. total $(29,61 \%)$ entre os solos estudados, sendo, portanto, solo em que ocorre a menor sorção do herbicida $\left(3,63 \mathrm{~mL} \mathrm{~g}^{-1}\right)$.

Desse modo, supõe-se que o no solo 1 o herbicida halossulfurom metílico apresente uma maior mobilidade, podendo sofrer processos como runoff e percolação no solo, atingindo assim corpos hídricos superficiais e subterrâneos. Pelo tempo de meia-vida do herbicida ser moderado, a possibilidade de contaminação de corpos hídricos superficiais na proximidade do solo 1 é maior do que os corpos hídricos subterrâneos. Já para o solo que apresentou maior $\mathrm{K}_{\mathrm{d}}$, no caso o solo 4, a permanência do halossulfurom metílico no solo é maior, diminuindo a possibilidade de contaminação de águas superficiais e subterrâneas pelos processos de runoff e percolação do composto.

Em vista disso, faz-se necessário uma maior atenção nas propriedades dos solos, especialmente qualidade e quantidade de matéria orgânica e argilominerais presentes em cada solo, para prever a contaminação de solos e corpos de águas próximos a aplicação do herbicida halossulfurom metílico. Além do mais, as propriedades físico-químicas do próprio herbicida também devem ser consideradas, como capacidade de sorção em solos, $\mathrm{pK}_{\mathrm{a}}$ e tempo de meia vida.

\section{CONCLUSÃO}

Considerando a análise de $\mathrm{K}_{\mathrm{d}}$ e $\mathrm{K}_{\mathrm{oc}}$ do halossulfurom metílico em todos os quatro solos amostrados, pode-se observar uma maior adsorção deste herbicida no solo 4, porém um nível de adsorção moderado, possivelmente devido à qualidade e tipo de matéria orgânica e argila que este solo apresenta. 0 solo 1 , apesar de possuir maior teor de matéria orgânica, foi o que menos adsorveu o herbicida. Os solos 2 e 3 apresentaram adsorção intermediária. 0 halossulfurom metílico apresenta correlação positiva com o teor de C.T.C. total e argila dos solos e uma correlação negativa com matéria orgânica e pH. Assim, é possível que o herbicida seja mais adsorvido nos componentes inorgânicos do solo do que nos orgânicos dos solos.

\section{AGRADECIMENTOS}

Essa pesquisa recebeu apoio da FAPESP (06/54764-8) e Unesp (IBILCE).

\section{REFERÊNCIAS BIBLIOGRÁFICAS}




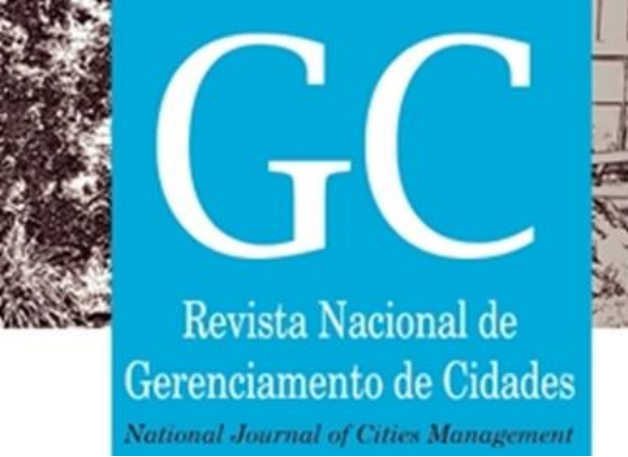

ALISTER, Claudio, A.; ARAYA, Manuel A.; KOGAN, Marcelo. Adsorption and desorption variability of four herbicides used in paddy rice production. J. Environ. Sci. Health. B., 46, 1, 62-68, 2011.

AYANO, Eri; KANAZAWA, Kanazawa; ANDOA, Masanori; NISHIMURA, Tetsuji. Determination and quantitation of sulfonylurea and urea herbicides in water samples using liquid chromatography with electrospray ionization mass spectrometric detection. Analytica Chimica Acta, 507, 211-2018, 2004.

CABRERA, A.; TRIGO, C.; COX, L.; CELIS, R.; HERMOSIN, M.C.; Cornejo, J.; Koskinen, W.C.. Sorption of the herbicide aminocyclopyrachlor by cation-modified clay minerals. Eur. J. Soil Sci., 63, 5, 694-700, 2012

CAMARGO, Otávio, A.; MONIZ, Antonio C.; JORGE, José A.; VALADARES, José. M. A. S. Métodos de Análise Química, Mineralógica e Física de Solos do Instituto Agronômico de Campinas. Campinas, Instituto Agronômico, 2009. 77 p. (Boletim técnico, 106, Edição revista e atualizada).

CRITTER, Silvana A.M.; AIROLDI, Claudio. Adsorption-desorption processes of calcium on Brazilian soils. Geoderma, 111, 57, 2003.

FARENHORST, A.; MCQUEEN, D.A.R.; SAIYED, I.; HILDERBRAND, C.; LI, S.;LOBB, D.A.; MESSING, P.; SCHUMACHER, T.E.; PAPIERNIK, S.K.; LINDSTROM, M.J.. Variations in soil properties and herbicide sorption coefficients with depth in relation to PRZM (pesticide root zone model) calculations. Geoderma, 150, 3-4, 267-277, 2009.

FILIPE, Olga M.S.; COSTA, Carina A.E.; VIDAL, Maria M.; SANTOS, Eduarda B.H. Influence of soil copper content on the kinetics of thiram adsorption and on thiram leachability from soils. Chemosphere, 90, 2, 432-440, 2013.

GAULTIER, Jeanette; FARENHORST, Annemieke; KIM, Sung, M.; SAIYED, Ibrahim; MESSING, Paul; CESSNA, Allan J.; GLOZIER, Nancy E. Sorption-Desorption of 2,4-Dichlorophenoxyacetic Acid by Wetland Sediments. Wetlands, 29, 3, 837-844, 2009.

HIRAYAMA, Yuichi; OHMICHI, Masayoshi; TATSUMOTO, Hideki, Simple and Rapid Determination of Golf Course Pesticides by In-Tube Solid-Phase Microextraction Coupled with Liquid Chromatography. J. Health Sci., 51, 526-532, 2005.

HOMENZUKA, Shozo \& YAMAMOTO Akira. Degradation of the herbicide halosulfuron-methyl in two soils under different environmental conditions. J. Pestic. Sci. 22:282-287. 1997.

IBAMA - Instituto brasileiro de meio ambiente e dos recursos renováveis. Manual de pesticidas para a avaliação da ecotoxidade de agentes químicos. 2.ed. Brasília, IBAMA, 1990, parte E. (não paginado).

ISHIMITSU, Susumu; KAIHARA, Akiko; YOSHI, Kimihiko; TSUMURA, Yukari; NAKAMURA, Yumiko; TONOGAI, Yasuhide. Simultaneous Determination of Azimsulfuron, Flazasulfuron and Halosulfuron-methyl in Grains, Seeds, Vegetables and Fruits by HPLC. J. Health Sci., 48, 335-340, 2002.

KIEHL, E. J. Manual de Edafologia, Relações Solo - Plantas. São Paulo. Editora Agronômica Ceres, 1979, p.264.

LEMOS, R.C. \& SANTOS, R.D. Manual de descrição e coleta de solo no campo. Sociedade Brasileira de Ciência do Solo - Centro Nacional de Pesquisa de Solos, Campinas-SP, 1996, p. 83.

MULLER, John, ABDELOUAS \& Abdesselam. Abdelouas. Sorption of selenite in a multi-component system using the "dialysismembrane" method. Appl. Geochem., 27, 12, 2524-2532, 2012.

OLIVEIRA, José B. Solos do Estado de São Paulo: descrição das classes registradas no mapa pedológico. Campinas, Instituto Agronômico, 1999. 112p. (Boletim Científico 45). 
